\title{
The Logical Structure of Binding
}

\author{
António Branco \\ DFKI and Univ. of Lisbon \\ Dep. Informática, Fac. Ciências, Campo Grande, 1700 Lisboa, Portugal \\ Antonio.Branco@di.fc.ul.pt
}

\begin{abstract}
A logical recasting of Binding Theory is performed as an enhancing step for the purpose of its full and lean declarative implementation. A new insight on sentential anaphoric processes is presented which may suggestively be captured by the slogan binding conditions are the effect of phase quantification on the universe of discourse referents.
\end{abstract}

\section{Introduction}

Due to its central role in natural language and its intriguing properties, reference and anaphor resolution has been a central topic for NLP research. Given the intensive attention devoted to this subject, it can however be said that sentential anaphor processing has been quite overlooked, when compared to the amount of research effort put in tackling non sentential anaphoric dependencies. This tends to be so because there seems to be a more or less implicit assumption that no substantial difference exists between the two processes 1 .

While this may be arguably true for the heuristics involved in picking out a given antecedent from a list of suitable candidates, a more subtle point asks itself to be made when we focus on the syntactic conditions which sentential anaphoric relations comply with, but from which non sentential ones are exempt.

In theoretical linguistics these grammatical conditions are grouped under the heading of Binding Theory. In computational linguistics however, though there have been a few papers directly concerned with the implementation of this theory, mainstream research tends to disregard its conceptual, grammatical or practical modularity. When it comes to define the algorithm for setting up the list of suitable candidates from which the antecedent should be chosen, binding conditions, holding just at the sentential level, are most often put on a par with any other kind of conditions, morphological, semantic, pragmatic, etc. which hold for anaphoric relations at both sentential and non sentential level

The interesting point to be made in this connection is that, if the modularity of grammatical knowledge is to be ensured in a sound reference resolution system. more attention should be paid to previous attempts of implementing Binding Theory. It would then become evident that this theory, in its current formulation, appears as a piece of formalised grammatical knowledge which however escapes a fuhl and lean declarative implementation.

In fact, implementation efforts concerning Binding Theory $y^{2}$ bring to light what tend to be eclipsed by mainstream clean theoretical formulations of it. Behind the apparent declarative aspect of its definition under the form of a set of binding principles (plus definitions of associated concepts, e.g. o-command, o-bound, local

'As entry points into bibliography vd References in Grosz et al. (95) and Botley et al. (96).

${ }^{2}$ Vd. Chomsky(81), Correa(88), Ingria et al (89), Fong (90), Giorgi et al. (90), Pianesi (91). domain, etc.), there is a set of procedures which turn out to be an essential part of the theory: after parsing being completed, (i) indexation: assign indices to NPs; (ii) filtering: store the indexed tree if the indexation respects binding principles, reject otherwise; (iii) recursion: repeat (i) with a new assignment until all possible assignments are exhausted.

This sort of resistance to declarative encompassing is also apparent when one considers how Binding Theory is handled in grammatical theories developed on top of constraint based formalisms and particularly concerned with computational implementability, like LFG or HPSG.

As to HPSG, it has passed quite unnoticed that its Binding Theory is the only piece of the grammar fragment not encoded in its own formalism. In the Appendix of the foundational book (Pollard and Sag (94)), where the fragment of grammar developed along its $400 \mathrm{pp}$. is encoded in the adopted formalism, Binding Theory escapes such encoding. Bredenkamp (96) and Backofen $e t$ al. (96) subsequent elaboration on this issue implied that some kind of essential limitation of the formalism might have been reached and that HPSG Binding Theory is still waiting to be accommodated into HPSG grammars.

As to the LFG formulation of Binding Theory, it requires the integration of inside-out equations, a special purpose extension to the general declarative formalism. And even though initial scepticism about their tractability was dissipated by Kaplan and Maxwell (88), the recent survey of Backofen et al. (96) reports that no implemented formalism, and no implemented grammar, is known to handle LFG Binding Theory.

In this connection the central aim of the research to be presented here is to render possible a lean declarative implementation of Binding Theory in constraint based formalisms without resorting to specific complex mechanisms. This involves two steps. First, as a sort of enhancing step back, a new account of Binding Theory is set up. Second, by the exhibition of an example the new shape of the theory is shown to support full declarative implementation in basic HPSG formalism. Due to space constraints, this paper is mostly concerned with the first while the latter receives just a rough sketch in last section, being developed in future papers.

\section{Preliminaries}

\subsection{The Square of Opposition}

Recent cross linguistic research, e.g. Xue, Pollard and Sag (94) and Branco and Marrafa (97), has shown that the binding ability of long-distance reflexives is not reducible to recursive concatenation of short distance relations, as it has been assumed in GB accounts, but that it is ruled by a fourth binding principle:

\section{(1) Principle Z}

An o-commanded anaphoric pronoun must be o-bound. 
(2) $\mathrm{Z}$

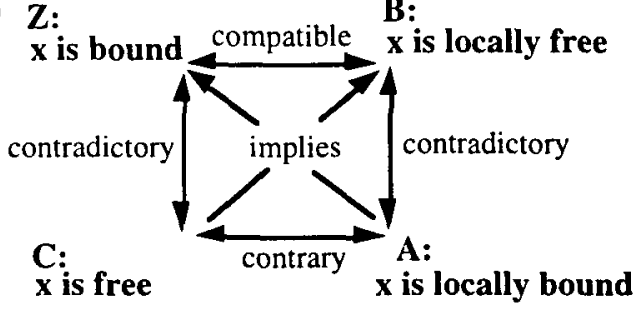

This new perspective on long-distance reflexives had an important impact in the whole shape of Binding Theory Branco and Marrafa noted still that the four principles can be arranged in a classical Aristotelian square of oppositions, as in (2). This suggests that the Binding Theory may have an unsuspected underlying quantificational structure. The present paper aims at showing that there is such structure and at determining its basic lines.

\subsection{Phase Quantification}

Barwise and Cooper (81) seminal work gave rise to a fruitful research tradition where Generalised Quantifier Theory has been applied to the analysis of natural language quantification. These authors suggested that a universal characterisation of NL nominal quantification could be formally given by means of formal properties defined in that theory. The property "to live on" was postulated as being the most prominent one, admittedly constituting the common specific nature of all nominal quantifiers.

Later, Loebner (87) suggested a criterion to ascertain the quantificational nature of natural language expressions in general. That is the property that, for a one place second order operator $Q$ expressed by a given expression, there be a corresponding dual operator Q $\sim$.

This duality based perspective on the essence of natural language quantification permitted to extend quantification well beyond the classic cases of nominal quantification supported by the determiners all, some, most, many, etc., namely by covering also the realms of temporality and possibility. Moreover, items like stilli already, and others (enough/too, scaling adjectives, many/few, etc.) though they do not lend themselves to be straightforwardly analysed in terms of set quantification, they can also be arranged in a square of duality. The formalization of the semantics of these aspectual items by Loebner led to the enlarging of the notion of quantification through the introduction of the new concept of phase quantification.

He noted that still and already express duals and that they are corners of a square of duality. Let $\mathrm{P}$ be "she is asleep" and $\sim \mathrm{P}$ "she is awake", durative propositions which are the arguments of the semantic operators corresponding to already and still. Then:

(3) She is already asleep iff

it is not the case that she is still awake.

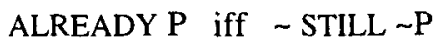

Further similar tests can be made in order to show that these aspectual items enter the following square of duality:

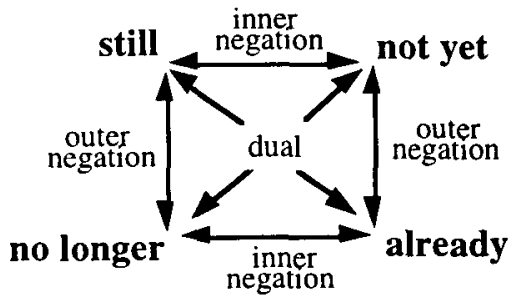

In order to get a formalization of (4), Loebner noted that already should be taken as conveying the information that there is a phase of not- $P$ which has started before a given reference time to and might be followed by at most one phase $\mathrm{P}$ which reaches till $t 0$. This can be displayed in a time axis by means of the diagram in (5).

(5)
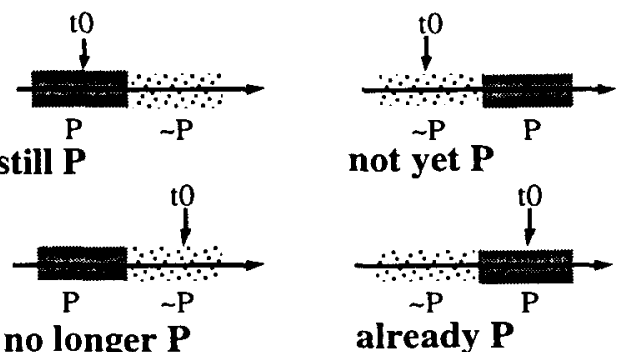

no longer $\mathbf{P}$

already $P$

Similar diagrams for the meaning of the other aspectual phase quantifiers of this square of duality are easily interpretable. Inner negation results in exchanging the positive and the negative semiphases, while outer negation concerns the decision whether the parameter $t 0$ falls into the first or the second semiphase.

Phase quantifiers in general (already, scaling adjectives, etc.) were thus characterised as requiring two ingredients: (i) a property $P$, which defines a positive phase in a sequence of two opposite phases; (ii) a parameter point. The four types of quantifiers just differ in presupposing that either the positive or the negative semiphase comes first and in stating that the parameter point falls into the first or into the second semiphase.

Next Loebner showed that the semantics of phase quantifiers sketched in the diagrams above can be formalised in such a way that a square of duality formed by the generalised quantifiers $\lambda X$.some' $(D, X) /$ $\lambda X$.every $(D, X)$ turns out to be subjacent to the square of duality of already/still. In order to do it, he just needed the auxiliary notion of starting point of the relevant semiphase. This is rendered as the infimum of the set of the closest predecessors of the parameter point $p t$ which form an uninterrupted linear sequence with property $\mathrm{P}$, or $\sim \mathrm{P}$ (termed GSI(R,pt) by Loebner):

(6) $\quad \operatorname{GSI}(\mathrm{R}, \mathrm{pt})=\mathrm{df} \inf \{\mathrm{x} \mid \mathrm{x}<\mathrm{pt} \& \mathrm{R}(\mathrm{x}) \&$

$$
\forall \mathrm{y}(\mathrm{x}<\mathrm{y} \leq \mathrm{pt} \& \mathrm{R}(\mathrm{y}) \rightarrow \forall \mathrm{z}(\mathrm{x}<\mathrm{z}<\mathrm{y} \rightarrow \mathrm{R}(\mathrm{z})))\}
$$

The semantics of the four phase quantifiers above can then be rendered in the following way, making $p t=t 0$ for the parameter point and $\mathrm{R}=\mathrm{P}$ or $\mathrm{R}=-\mathrm{P}$ :

(7) still: $\quad \lambda$ P.every' $(\lambda x \cdot(G S I(P, a)<x \leq t 0), P)$

already: $\quad \lambda P . s o m e '(\lambda x .(G S I(\sim P, a)<x \leq t 0), P)$

not yet: $\lambda$ P.no' $(\lambda x .(G S I(\sim P, a)<\mathrm{x} \leq \mathrm{t} 0), \mathrm{P})$

nolonger: $\lambda$ P.not every' $(\lambda x$. $(G S I(P, a)<x \leq t 0), P)$

\section{The Logic of Binding}

Taking Loebner's view on quantification, our goal in this section is to make apparent the quantificational structure of binding by showing that on a par with the square of opposition of (2) binding principles form a square of duality. We are going thus to argue that binding principles are but the reflex of the phase quantificational nature of corresponding nominal expressions: reflexives, pronouns long-distance reflexives and $\mathrm{R}$-expressions will be shown to express phase quantifiers acting on the grammatical obliqueness axis. 


\subsection{Phase quantification ingredients}

In order to show that the above referred nominals express phase quantifiers the relevant components involved in phase quantification should be identified.

The relevant scale here is not the continuous linear order of moments of time, as for still/already, but a discrete partial order made of discourse referents (cf. DRT) arranged according to the relative obliqueness of grammatical functions. Note that in multiclausal constructions there is the corresponding subordination of different clausal obliqueness hierarchies (for the sake of comparability with diagrams (5) involving time arrow, Hasse diagrams for obliqueness are displayed with a turn of $90^{\circ}$ right)

(8) Kim said Lee saw Max.

$$
\mathrm{k}-\mathrm{l}-\mathrm{m}
$$

Note also that the relation "less oblique than" may not be linear:

(9)Kim said Lee, who saw Max, hit Norma.

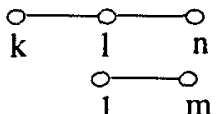

The sequence of two opposite semiphases is defined by a property $P$. Contrarily to what happens with already, where operator (quantifier) and operand (durative proposition) are rendered by different expressions, in binding phase quantification the operand $P$ ' is also contributed by the nominal expressing the operator, i.e. expressing the binding phase quantifier.

For a given nominal $\mathrm{N}, \mathrm{P}$ is determined by the relative position of $\mathrm{N}$ in the "scale". For a discourse referent $r$ corresponding to $\mathrm{N}$, semiphase $\mathrm{P}$ is a linear stretch containing only elements that are less than or equal to $r$ in the obliqueness order, that is discourse referents corresponding to nominals o-commanding $\mathrm{N}$. Moreover, if semiphase $P$ is presupposed to precede semiphase $-P, P$ is such that the last successor in it is local wrt to $r$; and if semiphase $\sim P$ is presupposed to precedes semiphase $P, P$ is such that the first predecessor in it is local wrt to $r$. In both cases the closest $\mathrm{P}$ neighbour of semiphase $\sim \mathrm{P}$ has to be local wrt $r$, where the notion of locality has the usual sense given in the definition of binding principles:

\section{(10) $\mathrm{P}(\mathrm{x})$ iff $_{\text {def }} \mathrm{x} \leq \mathrm{r} \& \forall \mathrm{y}[(\sim \mathrm{P}(\mathrm{y}) \&$ \\ $(\mathrm{x}-<\mathrm{y}$ or $\mathrm{y}-<\mathrm{x})) \rightarrow \mathrm{x}$ is local wrt $\mathrm{r}]$}

As to the parameter point, in binding phase quantification, it is the discourse referent $a$ which is the antecedent of $r$.

\subsection{Binding phase quantifiers}

We can now formalise phase quantification subjacent to nominals. Let us start with an anaphoric expression $\mathrm{N}$ like himself.

(11)Kim said Lee thinks $\operatorname{Max}_{i}$ hit himself $i$.

${ }^{*}$ Kim said Lee $i$ thinks Max hit himself $i$.

$\mathbf{Q}_{\mathbf{A}}: \lambda$ P.some' $(\lambda x .(\mathrm{GSI}(\sim \mathrm{P}, \mathrm{a})<\mathrm{x} \leq \mathrm{a}), \mathrm{P})$

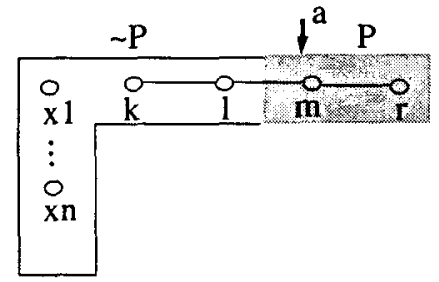

$\mathrm{N}$ can thus be interpreted as presupposing that a semiphase $\sim P$ precedes a semiphase $P$ and requiring that the parameter point occurs in the latter, that is, the antecedent $a$ is to be found in semiphase $\mathrm{P}$ among the discourse referents corresponding to the local ocommanders of $r$, the disc referent corresponding to $\mathrm{N}^{3}$. This is captured by the definition of the phase quantifier $\mathrm{Q}_{A}$. Satisfaction of $\mathrm{QA}_{A}(\mathrm{P})$ obtains iff between the bottom of the uninterrupted linear sequence $\sim \mathrm{P}$ most close to the parameter point/antecedent $a$ and $a$ inclusive there is at least one discourse referent in P. Given P.P, this amounts to requiring that $a$ be in $\mathrm{P}$, and that $a$ be a local ocommander of $r^{3}$

Next, it is then easy to see how the phase quantificational force of a pronominal expression $\mathrm{N}$ should be formalised:

(12) ${ }^{*}$ Kim said Lee thinks $\operatorname{Max}_{i}$ hit $\operatorname{him}_{i}$. Kim said Lee $i$ thinks Max hit him $i$.

$$
\text { QB: } \lambda \text { P.no' }(\lambda x .(G S I(\sim P, a)<x \leq a), P)
$$

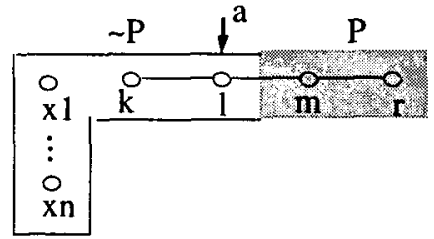

Here the parameter point $a$ occurs in semiphase $\sim P$, which amounts to the antecedent being picked outside the set of local o-commanders. $\mathrm{OB}_{\mathrm{B}}(\mathrm{P})$ is satisfied iff no discourse referent between the bottom of the uninterrupted linear sequence $\sim \mathrm{P}$ more close to the parameter point/ antecedent $a$ and $a$ inclusive is in P. Given $\sim$ P.P, this amounts to requiring that $a$ be in semiphase $\sim \mathrm{P}$, and that $a$ be not a local o-commander of $r$.

Like in diagram of (11) $\sim \mathrm{P}$ is taken here as the complement set of $P$. All discourse referents which are not local o-commanders of $r$ are in it, either ocommanding $r$ or not. Notice that set $\sim \mathrm{P}$ includes also discourse referents $x_{1} \ldots x_{n}$ introduced by previous sentences or the extra-linguistic context, which in constructions similar to $(1 / 2) \mathrm{b}$. accounts for possible deictic readings of the pronoun. Below, when studying R-expressions, we will see why the possible non linearity of the obliqueness order will led us to consider that $\sim P$ is slightly more complex than just the complement set of $P$.

Coming now to long-distance reflexives, ruled by the fourth binding principle in (1), we get the following formalisation:

(13)[O amigo de $\mathrm{Kim}]_{i}$ disse que ele próprio $i$ acha que Lee viu Max. (Portuguese) [Kim's friend] $i$ said LDR $i$ thinks Lee saw Max.

*[O amigo de $\mathrm{Kim}_{i}$ ] disse que ele próprio $i$ acha que Lee viu Max.

[Kim's $i$ friend] said $\mathrm{LDR}_{i}$ thinks Lee saw Max.

$$
\text { QZ: } \lambda \text { P.every' }(\lambda x .(G S I(P, a)<x \leq a), P)
$$

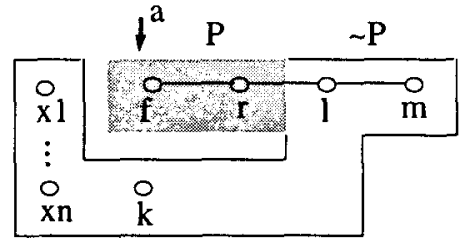

${ }^{3}$ For the sake of simplicity, agreement requirements between $\mathrm{N}$ and its antecedent are overlooked here. 
Here, like for short-distance reflexives in (11), $a$ is required to occur in $\mathrm{P}$ though the presupposition now is that semiphase $\mathrm{P}$ is followed by semiphase $\sim \mathrm{P}$. Taking into account the definition of $P$ in (10), the antecedent of $\mathrm{N}$ is thus required to be an o-commander (local or not) of $N$. The semantics of phase quantifier QZ is such that, for QZ(P) to be satisfied, between the bottom of the uninterrupted linear sequence $P$ more close to the parameter point/antecedent $a$ and $a$ inclusive every discourse referent is in $P$. This amounts to requiring that $a$ be in semiphase $\mathrm{P}$, and that $a$ be an o-commander of $r$.

Finally R-expressions call to be formalised as the fourth phase quantifier of $(7)$ :

(14) [Kim's $i$ friend] said $\mathrm{Kim}_{i}$ thinks Lee saw Max.

* [Kim's friend $]_{i}$ said $\mathrm{Kim}_{i}$ thinks Lee saw Max.

QC: $\lambda$ P.not every' $(\lambda x .(G S I(P, a)<x \leq a), P)$

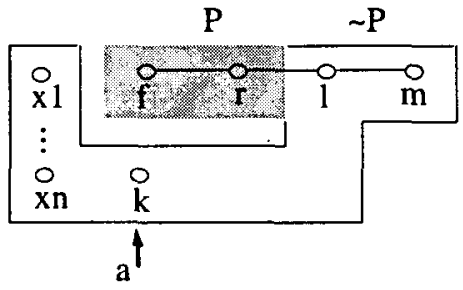

The parameter point $a$ is required to occur in $\sim \mathrm{P}$, which means that $a$ cannot be an o-commander (local or not) of $r$. This renders the same condition as expressed by Principle C, that R-expressions be free, though it also encodes an uncommon assumption about the referential autonomy of R-expressions. Here, like for other more obvious dependent reference nominals, the interpretation of R-expressions is taken as being dependent on the interpretation of other expressions or on the salience of discourse referents made available by the communicative context. Taking an extreme example in order to support the plausibility of this view and awkwardly abbreviate a deep philosophical discussion, one should notice that even a proper name is not a unique label of a given individual, once knowing who is the person called John (out of those we know that are named John) depends on the context. Note that like in previous diagrams, $-\mathbf{P}$ is taken in (14) just as the complement set of P. However, QC asks finally for a serious ponderation of this and a more accurate definition of $\sim \mathrm{P}$ for phase quantification in non linear orders, where it is possible that not all elements are comparable.

For $\mathrm{OC}(\mathrm{P})$ to be satisfied, between the bottom of $\mathrm{P}$ and the parameter point/ antecedent $a$ inclusive not every discourse referent is in $\mathrm{P}$. Since we have here the presupposition P. P, and given $P$ is an uninterrupted linear sequence, this would amount to requiring that $a$ be in $\sim$ P.

It is worth noting then that if we keep $\sim \mathrm{P}$ simply as the complement set of $P$, the interpretation of $R$ expressions is however not adequately predicted by QC(P).

(15) John said $\mathrm{Kim}_{j}$ thinks Lee saw Max.

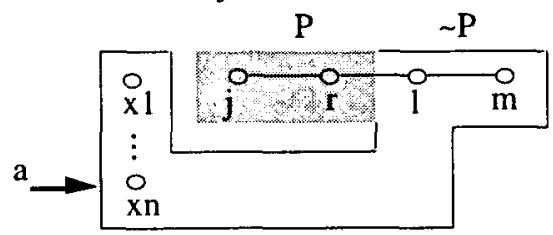

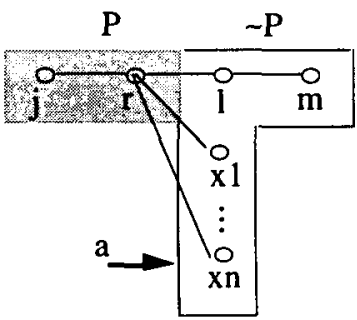

Let $D$ be $\{x$ : GSI $(P, a)<x \leq a\}$, the domain of QC. Taking (15)b., it is easy to check that in constructions like (15) a. D is always empty. In fact, it is not the case that $G S I(P, a) \leq a$ as $a=x T$ is not comparable to any element of $\mathrm{P}$, and a fortiori it is not comparable to the bottom of $P$. Consequently, every' $(D, P)$ is trivially true whatever discourse referent $\mathrm{x} n$ we take as antecedent for $r$, and not every'(D,P) is trivially false. The interpretation of (15)a. sketched in (15)b. would thus be incorrectly ruled out.

What these considerations seem then to suggest is that, when phase quantification operates on non linear orders, negation of the operand $\mathrm{P}$ is slightly more complex than simple Boolean negation rendering the complement set. We are thus taught that negation of $\mathrm{P}$ involves also the lifting of the complement set of $P$, $\mathrm{P}_{\perp}$, with $\perp$ equal to $r$ the top of $\mathrm{P}$, when $\mathrm{P}$. $\sim \mathrm{P}^{4}$. It is easy to check with diagram (15)c. that this specification of $\sim \mathrm{P}$ makes it possible to satisfy $\mathrm{QC}(\mathrm{P})$ in exactly the correct constructions.

\subsection{The Binding Square of Duality}

Following Loebner's claim that logical duality is the cardinal property to recognise the quantificational character of natural language expressions, we are thus led to the view that the interpretation of non quantificational definite nominals is ruled by their phase quantificational force over the obliqueness order. Since the defining formulas of binding quantifiers result from (7) just by assigning $\mathrm{P}$ the definition in (10) and taking the parameter point $p t$ to be the antecedent $a$, it is with no surprise that we get the following square of duality for binding quantifiers:

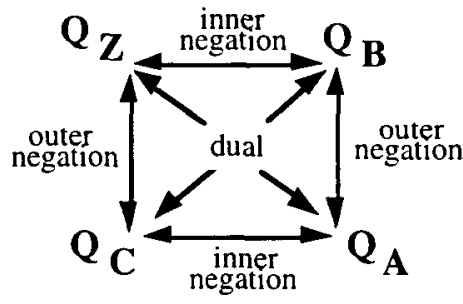

\section{Consequences}

This new conception of binding seems to have important consequences not only in terms of the understanding of dependent reference mechanisms captured by Binding Theory but also in terms of our conception of generalised quantification in natural language, of the twofold semantic capacity of nominal expressions, referential and quantificational, and maybe even of the nature of grammar devices. Here we cannot do but to limit ourselves to hint how a few central issues usually associated to binding are handled under this new viewpoint, before we proceed to briefly consider its consequences for the implementation of Binding Theory in constraint based grammars.

\subsection{Further insights into binding...}

Parameterization It is well known that though binding principles are assumed to hold universally in all languages, final "grammatical geometry" between 
nominals and their antecedents may be different from language to language.

Dalrymple (93) pointed out that this is due to language specific conditions impinging (i) on the eligibility of the antecedent (whether it is a Subject or not) and (ii) the range of the local domain (whether it is finite, tensed, etc.). As to (i), Branco and Marrafa (97) showed that it is a consequence of a lexical property of the predicates, whose obliqueness hierarchy may be either linear or non linear. As to (ii), this variation may be accommodated in the definition of property $P$ in $(10)$, in particular in the definition of "local wrt to $r$ ", to be provide for each particular language. Both solutions are perfectly confluent with the $0 \mathrm{G}$ standpoint that binding variations across language are the result of parameterization

Lexical gaps ${ }^{4}$ It is also well known that although the four binding principles are claimed to be universal there are languages which have not all the corresponding four type of nominals. For instance, English is not known to have long-distance reflexives. The answer for this becomes now quite simple: like what happens in other squares of duality, it is possible that not every corner of the square is lexicalized. Loebner (87) discusses at length the issue. In English, for instance, it is noted that the square of duality concerning deontic possibility involving right happens to have only two lexicalized corners, right and duty.

Exemption and logophoricity Also worth considering here is the borderline case where the maximum shrink of semiphase $P$ occurs, i.e. when $P$ is the singleton whose sole element is $r$, the discourse referent whose interpretation is to be anchored by finding an antecedent for it.

Given the definition of binding phase quantifiers, the maximum shrink of $P$ into a singleton affects significantly only the quantifiers where the parameter point/antecedent $a$ is to be found in P, namely $\mathrm{QA}_{\mathrm{A}}$ and Q7. In these cases, for $a$ to be in $P$ and the quantification to be satisfied, $a$ can only be $r, r$ being thus its own antecedent. Consequently, although the quantification is satisfied, a "meaningful" anchoring of the discourse referent $r$ is still to be accomplished since by the sole effect of quantification satisfaction $r$ is just anchored to itself. Admittedly, an overarching interpretability requirement imposes that the significant anchoring of nominals be consummated, which induces in present case an exceptional logophoric effect: for the anaphor (short or longdistance) to be interpreted, and given that satisfaction of its binding constraint is ensured, it should thus freely find an antecedent outside any specific restriction.

This constitutes thus an explanation for the exemption restrictions in the definitions of Principles $A$ and $Z$ and so called logophoric effects associated to exempt anaphors. Restrictions which appeared until now to be mere stipulations receive in this approach a principled justification.

\section{2 ... for a lean implementation}

The new conception of Binding Theory presented in this paper is currently being integrated in an HPSG grammar implemented in ProFIT 1.54. Space limits restrict us here to a very brief rationale of that implementation, which will be fully presented in future papers.

The interesting point to note in this connection is that the new insight into binding phenomena elicited by the discovery of their quantificational nature seems to constitute a breakthrough for the desideratum of giving Binding Theory a lean declarative implementation. Adopting a principle based semantics in line with Frank and Reyle (95), the central goal is not anymore

${ }^{4}$ Though it is empirically not necessary, for the sake of uniformity, when $-P . P$, the order-theoretic dual of this specification of $\rightarrow P$ can be assumed. to filter coindexations between NPs in post-processing but rather to identify the relevant sets of discourse referents against which satisfation of the binding phase quantification expressed by NPs is checked.

In practical terms that involves first collecting discourse referents into set values of specific features, requiring a minor extension to HPSG feature declaration. Second, given the possible non local nature of the elements of a given set, in order to avoid termination problems some mechanism of delaying constraint satisfaction has to be ensured.

\section{Conclusions}

The research reported here present a cogent argument for the quantificational nature of sentential dependent reference relations among nominals. This radically new conception of binding appears as a decisive step towards a full lean declarative encompassing of Binding Theory in constraint based grammars. It may have also opened new intriguing girections for the research on natural language generalised quantification, on the apparent twofold semantic capacity of nominals, referential and quantificational, or on the nature of grammar devices.

\section{Acknowledgements}

Special thanks are due to Palmira Marrafa and Hans Uszkoreit for their advice and discussion and to Berthold Crysmann for his detailed comments.

\section{References}

Chomsky (81), Lectures on Government and Binding, Foris, Dordrecht.

Correa (88), "A Binding Rule for Government-binding Parsing", COLING'88 Proceedings.

Backofen, Becker, Calder, Capstick, Dini, Dörre, Erbach, Estival, Manandhar, Mineur, van Noord, Oepen and Uszkoreit (96), Final Report of EAGLES Formalisms Working Group.

Barwise and Cooper (81), Generalized Quantifiers and Natural Language, $L \& P 4,159-219$.

Botley, Glass, McEnery and Wilson, eds. (96), Proceedings of Discourse Anaphora and Resolution Colloquium, Lancaster University.

Branco and Marrafa (97), "Long-Distance Reflexives and the Binding Square of Opposition", 4th International Conf. on HPSG.

Bredenkamp (96), Towards a Binding Theory for HPSG, PhD dissertation, Univ. of Essex.

Dalrymple (93), The Syntax of Anaphoric Binding, CSLI, Stanford.

Erbach (95), ProFIT 1.54 User's Guide, DFKI.

Fong (90), "Free Indexation: Combinatorial Analysis and a Compositional Algorithm", Proceedings of ACL Meeting, 105110.

Frank and Reyle (95), "Principle Based Sernantics for HPSG", Proceedings of EACL'95 Meeting.

Giorgi, Pianesi and Satta (90), "A Computational Approach to Binding Theory", Proceedings of COLING'90, 1-6.

Grosz, Joshi and Weinstein (95), "Centering: A Framework for Modelling the Local Coherence of Discourse", Computational Linguistics 21

Ingria and Stallard (89), A Computational Mechanism for Pronominal Reference, Proceedings of ACL Meeting, 262-271.

Kaplan and Maxwell (88), "An Algorithm for Functional Uncertainty", Proc. of COLING'88.

Loebner (87), "Quantification as a Major Module of Natural Language Semantics", in Croenendijk, Jongh and Stokhof, eds., Studies in DRT and the Theory of Generalized Quantifiers. Foris, Dordrecht.

Pianesi (91), "Indexing and Referential Dependencies within Binding Theory", Proceedings of EACL Conference, 39-44.

Pollard and Sag (94), Head-Driven Phrase Structure Grammar, CSLI, Stanford.

Xue, Pollard and Sag. (94), "A New Perspective on Chinese Ziji". Proceedings of the West Coast Conference on Formal Linguistics, vol. 13, CSLI, Stanford. 